\title{
Social transmission of food preferences among Norway rats by marking of food sites and by gustatory contact
}

\author{
K. N. LALAND and H. C. PLOTKIN \\ University College London, London, England
}

\begin{abstract}
Three experiments were conducted to investigate the social learning and transmission of food preferences by excretory marking among adult male Norway rats. The experiments extend our earlier findings that rats prefer to eat from a food bowl marked by the excretory deposits of conspecifics and that this mechanism can result in the communication and social learning of food preferences (Laland \& Plotkin, 1991). Here we investigate whether a tradition of food and food site preferences can become established by these means. Experiment 1 established that the residual cues deposited by rats lose their powers of communication as "markers" of food sites over a 72-h period. Experiment 2 showed that while a socially enhanced preference for one flavored diet could be transmitted from one animal to the next along a chain, it was unstable for an alternative diet. This suggests that social transmission may be more stable when it reinforces a prior preference than when it conflicts with one. In Experiment 3, the stability of socially transmitted food preferences was bolstered by the addition of a second process for the communication of diet preferences-namely, gustatory cues on the demonstrator's breath. This finding suggests that when a socially transmitted trait is mediated by more than one process, the processes may interact, and the diffusion is likely to be more stable.
\end{abstract}

In several field studies, researchers have reported the social transmission of foraging behaviors and food preferences among Norway rats (Calhoun, 1962; Gandolfi \& Parisi, 1973; Steiniger 1950). Such reports are supported by the findings from laboratory investigations of the passage of food and foraging information among rats (Galef, 1988; Galef \& Clarke, 1971; Galef \& Wigmore, 1983; Laland \& Plotkin, 1990). Galef and Heiber (1976) established that weanling Long-Evans rats preferred to eat from a food site in a section of the enclosure marked by the excretory deposits of a mature female conspecific rather than from a food site in an unmarked section. Galef and Beck (1985) found that Long-Evans rats can mark feeding sites, making them more attractive to weanling conspecifics than unmarked sites, and concluded that the communication of food site preferences could be mediated by olfactory cues surrounding particular feeding areas. In an earlier study, we investigated the communication and social learning of food preferences via excretory marking among adult male Norway rats (Laland \& Plotkin, 1991). We found that rats consistently preferred to eat from a

\footnotetext{
We are grateful to $\mathrm{C}$. Heyes of University College London and M. Morgan of Edinburgh University for their help and constructive advice throughout the study, and to $M$. Fanselow and the referees of this journal for helpful comments. This work was carried out under a Science and Engineering postgraduate studentship to K.N.L., who is now at the Department of Integrative Biology, University of California, Berkeley, CA 94720 . Correspondence should be addressed to H. C. Plotkin at the Department of Psychology, University College London, London WC1E 6BT, England.
}

food bowl marked by the excretory products of conspecifics than from an unmarked alternative, and that this mechanism could result in the communication and social learning of food preferences. We concluded that urine marking on and around the food site combines with fecal deposits to establish a stimulus complex that renders food sites attractive to rats, and results in the transmission of information about food preferences.

These experiments demonstrate that food site marking can be an effective mechanism for the passage of foodrelated information among Norway rats. If this process operates in natural populations, it is possible that food preferences may be socially transmitted by these means. Rats that feed from a site marked by the excretory deposits of conspecifics may themselves mark the site with their own products. Observations of the behavior of Norway rats both in the laboratory and in the wild suggest that they are continuously reinforcing their own scent marks and marking over other individuals' marks (Brown, 1985; Calhoun, 1962).

Following Curio, Ernst, and Vieth (1978), we have investigated social transmission in the laboratory by monitoring the stability of behaviors passed along a chain of animals (Laland \& Plotkin, 1990). We adopted this approach in the present study, extrapolating the design of our previous experiments to one in which each subject functions as the demonstrator for the next subject. In Experiments 2 and 3, we employed a design to investigate social transmission, in which we established a sequence of observer-demonstrator pairings with each observer becoming the demonstrator for the next animal, and we 
monitored the transmission of food preferences along a chain. But first, it was necessary to establish the time over which the cues that mark a food site remain active.

\section{EXPERIMENT 1}

To demonstrate social transmission along a chain of animals, it is important to establish that the period of time over which the cues remain active is substantially less than the duration of the chain. This will then rule out the possibility that all of the animals could be behaving in ways directed by the original scent marking. The scent-marking literature contains little information on the stability of rat pheromones. Estrous female urine has been found to be no longer more attractive to males than nonestrous female urine after 24 h (Lydell \& Doty, 1972). The markings of another species in the Muridae family, house mice (Mus musculus), tend to lose their marking efficacy after approximately $48 \mathrm{~h}$ (Brown, 1985; Jones \& Nowell, 1977). We expected the social odors of Norway rats to be equally transient in their effect. Consequently, in Experiment 1, we investigated whether the residual cues laid down by demonstrators would lose their powers of communication as markers of food sites over a 72-h period.

\section{Method}

Design. Animals were randomly allocated to one of five groups, four experimental conditions and one control condition. The animals in the control condition were placed for $24 \mathrm{~h}$ in a clean enclosure with two available food sites, each containing a different novel food, and their consumption of each diet was recorded. The animals in the experimental conditions were also placed in an enclosure containing two novel foods, but, for these animals, the enclosure had previously housed 4 demonstrator conspecifics that had eaten one of the novel foods from one of the sites. In two of these conditions, there was a 72-h delay between the demonstrators' being removed from the enclosure and the subject's being placed in it, and in the other two, there was no delay. The subjects in the no-delay experimental conditions were expected to consume more of the diet eaten by their demonstrators than were the subjects in the control group. If the residual cues were still active $72 \mathrm{~h}$ later, the animals in the delay experimental conditions would face a choice between a novel food at a soiled site and an alternative at a relatively unmarked site, and they would be expected to behave like the subjects in the other experimental groups. If, on the other hand, the residual cues left by the demonstrators should decay within $72 \mathrm{~h}$, the animals in the delay conditions would not be expected to behave significantly differently from the controls. In all conditions, the position of the bowls containing the two diets was varied systematically between subjects to counterbalance for position effects. The dependent variables were the amount of food eaten from each site and the number of feces and urine marks surrounding each site.

Subjects. These were 40 male rats of an agouti strain purchased from Harlan Olac Ltd., 10-12 weeks of age and weighing 179-219 g at test. An additional 128 identical animals acted as demonstrators.

Apparatus. The subjects were tested in a large U-shaped enclosure, measuring $170 \times 154 \times 36 \mathrm{~cm}$, with an aluminium frame and floor, clear Perspex walls, and a wire mesh roof. A white Perspex tray, measuring $32 \times 50 \mathrm{~cm}$, with a lip $3 \mathrm{~cm}$ high, was placed in each arm of the enclosure, upon which were placed the food bowls. During the demonstration (or marking) phase of the experiment, powdered standard rat diet (SDS, Lillico Ltd., Surrey, En- gland), flavored either with $1 \%$ (Schwartz) ground cinnamon or $2 \%$ (Safeway) cocoa, was available from a single stainless steel food bowl, $10 \mathrm{~cm}$ in diameter and with a 4-cm lip, placed centrally on one of the trays. To reduce spillage to a minimum, food bowls were only half filled. During test periods, a food bowl containing one diet was placed on one tray and an identical bowl containing the alternative diet was placed on the other. Water bottles were available in both of the arms of the enclosure. Both holding and experimental rooms were on a 12:12-h light:dark cycle (lights on at $1000 \mathrm{~h}$ ) and were maintained at a temperature $20^{\circ}-22^{\circ} \mathrm{C}$. Prior to experimentation, the subjects were housed in pairs in standard white Perspex enclosures with stainless steel wire roofs, measuring $25 \times 40 \times 20 \mathrm{~cm}$, with free access to food (Lillico Ltd. standard rat pellets) and water.

Procedure. For 7 days prior to the start of the experiment, all animals were handled daily, to prevent the experimental procedure from subjecting them to stress. In the control condition, we placed each subject for $24 \mathrm{~h}$ in a clean enclosure, with a food bowl on each of the trays, each bowl containing an equal amount of one of the two flavored diets. We then removed the subject and recorded the amount of diet eaten from each bowl. In the delay cinnamon demonstration condition, we placed 4 rats in the enclosure for $48 \mathrm{~h}$ and allowed them to eat from a single bowl containing the cinnamonflavored diet. We then removed the demonstrators, noted the distribution of feces and urine marks in the enclosure, and placed an equal amount of the cocoa-flavored diet in a bowl on the other tray. The enclosure was left in this state for $72 \mathrm{~h}$, and then we placed the subjects in the enclosure for $24 \mathrm{~h}$ and recorded their consumption of diet from each bowl. The procedure was exactly the same for the delay cocoa demonstration condition, except that the demonstrator animals were given a cocoa-flavored diet, and a bowl containing an equal amount of the cinnamon-flavored diet was subsequently placed on the other tray prior to the 72-h delay and the subjects' $24-h$ test. The procedure for the no-delay cinnamon demonstration and no-delay cocoa demonstration conditions was the same as that for the two delay conditions, except that there was no delay between removal of the demonstrators and the placement of the subject in the enclosure.

\section{Results and Discussion}

The mean amount of cinnamon and cocoa diet consumed by the subjects in each group is illustrated in Figure 1. The amount of cinnamon diet eaten varied significantly between the conditions $[F(4,35)=7.54, p<.01]$, with the subjects in the no-delay cinnamon demonstration group consuming significantly more of this diet $(M=19.21 \mathrm{~g})$ than did the other groups, and with the subjects in the no-delay cocoa demonstration group consuming significantly less $(M=5.89 \mathrm{~g})$ (Newman-Keuls, $p<.05)$. The subjects in the delay cinnamon demonstration and delay cocoa demonstration groups did not differ significantly from the controls in their cinnamon diet consumption $(M=11.58,13.26$, and $12.23 \mathrm{~g}$, respectively) (NewmanKeuls, $p>.05$ ). The amount of cocoa diet consumed also varied significantly between the conditions $[F(4,35)=$ $19.71, p<.01$ ], with the subjects in the no-delay cocoa demonstration group consuming significantly more of this $\operatorname{diet}(M=9.25 \mathrm{~g})$ than did the other groups, and with the subjects in the no-delay cinnamon demonstration group consuming less $(M=0.96 \mathrm{~g})$ (Newman-Keuls, $p<.05)$. The subjects in the delay cinnamon demonstration and delay cocoa demonstration groups did not differ significantly 


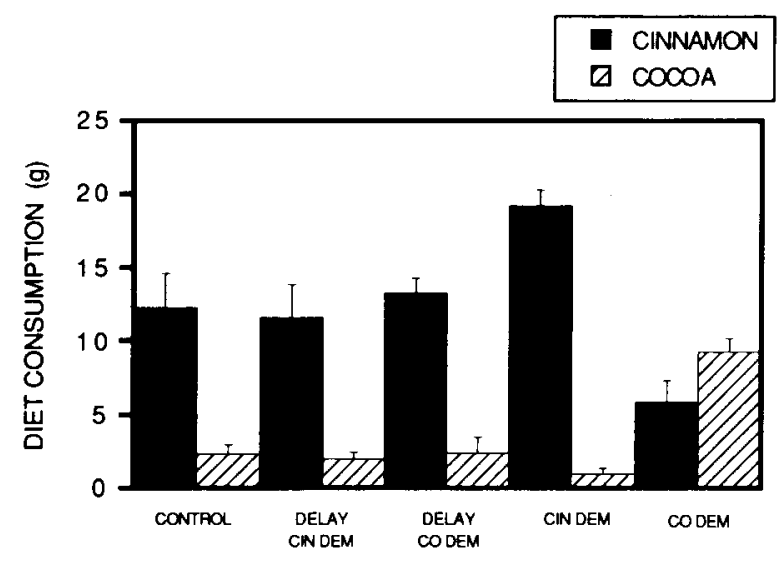

Figure 1. Mean and standard error food consumption in grams for each of the five groups in Experiment 1.

from the controls in their cocoa diet consumption $(M=$ $2.0,2.39$, and $2.33 \mathrm{~g}$, respectively) (Newman-Keuls, $p>$ $.05)$. There were no significant differences in the total amount of diet consumed by rats in the five conditions $[F(4,35)=2.01, p>.05]$.

Recordings of the excretory behavior of the demonstrators revealed that there were significantly more urine marks in the half of the enclosure containing the food bowl, discounting those on the bowl itself $(M=39.25)$, than in the other half $(M=11.63)$ [matched-sample $t(31)=3.2, p<.01]$, as well as more feces $(M=47.5$ and 21.23$)[t(31)=2.89, p<.01]$. There were no significant differences between demonstrators consuming cinnamon- and cocoa-flavored diets in the number of urine marks or fecal boli deposited, or in the patterns of deposition.

The results of this experiment provide strong evidence that communication of food preferences via excretory marking of food sites has occurred in the no-delay experimental groups, replicating our earlier findings (Laland \& Plotkin, 1991). There was no evidence for communication via excretory marking in the experimental groups with the 72-h delay. This finding lends support to the prediction that the communication properties of rats' residual products are vulnerable to decay, and that these cues become ineffective within a period of $72 \mathrm{~h}$ duration. An alternative explanation is that rats will only attend to olfactory cues that have been deposited relatively recently. This result suggests that the pertinent information for communication to take place is contained in olfactory or gustatory, rather than visual, cues. This is because visual cues would not be expected to decay over time, whereas chemical cues would dissipate rapidly.

\section{EXPERIMENT 2}

Experiment 1 established that the cues deposited by rats lose their powers of communication as markers of food sites over a 72 -h period. Thus, if an enhanced preference for one diet can be passed from one animal to another and then to a third along a transmission chain, and if that chain is tested for a period longer than 3 days, social transmission of that preference must have occurred. In other words, rats at intermediate links in the chain must not only be acquiring diet-related information from the excretory deposits of earlier demonstrators, but must themselves be acting as demonstrators by marking the food site with their own products. If social transmission does not occur or is unstable, the subjects that form the later links in the chain would not be expected to exhibit diet preferences different from those of animals with no social cues.

\section{Method}

Subjects and Apparatus. There were 240 rats of the same kind as in Experiment 1, tested in the same apparatus, with 64 animals in each experimental group and 48 controls.

Design and Procedure. There were three conditions: cinnamon and cocoa demonstration conditions and a control. In the cinnamon demonstration condition, we placed 4 rats in the enclosure for $48 \mathrm{~h}$ and allowed them to eat from a single bowl, counterbalanced for position, containing the cinnamon-flavored diet. We then removed these demonstrators, noted the distribution of feces and urine marks in the enclosure, and placed an equal amount of the cocoaflavored diet in a bowl on the other tray. Then we placed the 1st subject in the enclosure for $24 \mathrm{~h}$ and recorded its consumption of diet from each bowl. We then removed this animal, noted the distribution of urine marks and feces, and placed another subject in the soiled enclosure. The entire transmission chain procedure, with its eight transmission steps, was repeated eight times to give an $n=8$. The procedure was exactly the same for the cocoa demonstration condition, except that we gave the initial demonstrator animals a cocoa-flavored diet and subsequently placed a bowl containing an equal amount of the cinnamon-flavored diet on the other tray prior to the first subject's 24-h test. We produced a sufficient amount of the flavored diets initially to allow the levels in the bowls to be " topped up" if necessary. If the level of diet in a given food bowl was low, then, at the transition point between animals when the food bowls were weighed, we mixed in additional diet with that remaining. This spare diet was stored in open plastic bags. The control conditions had no initial demonstration. Here we placed each subject in a clean enclosure, with a food bowl on each of the trays, each bowl containing an equal amount of one of the flavored diets. The position of the bowls containing the two diets was varied systematically between subjects to counterbalance for position effects. After $24 \mathrm{~h}$, we removed the subject and recorded the amount of food eaten from each bowl. We then cleaned the enclosure, the trays, and the food bowls thoroughly, and replaced the food in each bowl, before placing another subject in the enclosure. The control chain procedure, with its eight independent steps, was repeated six times to give an $n=6$.

\section{Results and Discussion}

The amount of cinnamon diet consumed by subjects varied significantly between the groups $[F(2,152)=$ $15.22, p<.001$, with the subjects in both the cinnamon demonstration and the cocoa demonstration groups consuming more of this diet than did the controls $(M=$ 15.48 and $13.09 \mathrm{~g}$ vs. $9.93 \mathrm{~g}$, respectively) [cinnamon demonstration group, $F(1,152)=30.4, p<.01$; cocoa demonstration group, $F(1,152)=9.86, p<.01]$. The subjects in the cinnamon demonstration group also consumed significantly more cinnamon diet than did the sub- 
jects in the cocoa demonstration group (Newman-Keuls, $W_{2}=1.85, p<.05$ ). Although the subjects in the cocoa demonstration group consumed less cinnamon-flavored diet $(M=6.39 \mathrm{~g})$ at the first transmission step than did the controls, over the whole chain they consumed significantly more, and they exhibited an increasing trend in consumption $[F(1,152)=3.81, p<.05]$. There was no significant effect of stage in the transmission chain, and there was no interaction.

There were also significant differences in cocoa diet consumption among the groups $[F(2,152)=14.32, p<$ $.01]$, with the subjects in the cinnamon demonstration group consuming significantly less $(M=0.54 \mathrm{~g})$ than the subjects in the cocoa demonstration group $(M=3.14 \mathrm{~g})$ $[F(1,152)=21.06, p<.01]$ or the controls $(M=$ $3.33 \mathrm{~g})[F(1,152)=20.79, p<.01]$. In contrast, there was no difference between the cocoa demonstration and control conditions $[F(1,152)=0.1, p>.05]$. In addition, there was a significant main effect for transmission step $[F(7,152)=3.71, p<.01]$. The subjects in the cocoa demonstration group exhibited a decreasing trend in cocoa diet consumption across the chain $[F(1,152)=$ $17.85, p<.01]$, but the subjects in the cinnamon demonstration group showed no increasing trend in cocoa diet consumption $[F(1,152)=0.01, p>.05]$. The percentage cocoa diet consumption for each group across the chain is illustrated in Figure 2.

There were also differences between the groups in the total amount of diet consumed $[F(2,152)=7.41, p<$ $.01]$, with subjects in the cinnamon and cocoa demonstration groups eating more than the controls $(M=16.02$, 16.19 , and $13.09 \mathrm{~g}$, respectively) $[F(1,152)=14.77$, $p<.01]$.

Recordings of the excretory behavior of the demonstrators revealed that there were significantly more urine marks in the half of the enclosure containing the food bowl, discounting those on the bowl itself $(M=27.56)$ than in the other half $(M=11.19)$ [matched-sample $t(15)=7.56, p<.01]$, and more feces $(M=61.94$ and

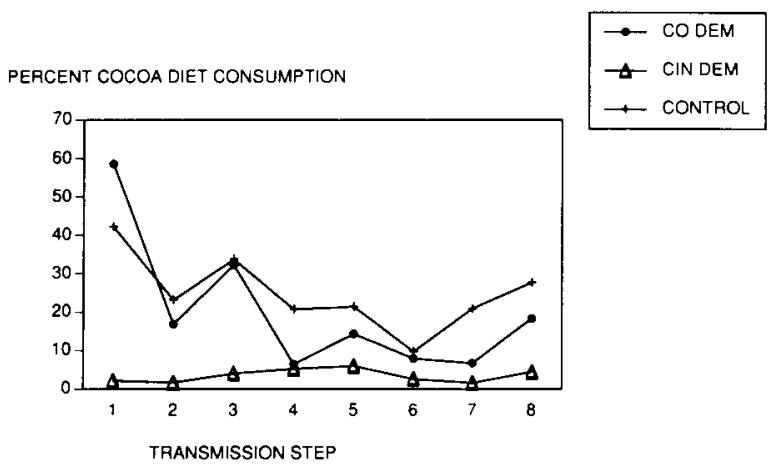

Figure 2. Experiment 2: Percent cocoa-flavored diet consumed by subjects in each group across the transmission chain. CO DEM and CIN DEM are the cocoa and cinnamon demonstration groups.
23.94) $[t(15)=2.89, p<.01]$. There were no significant differences between demonstrators consuming cinnamon- and cocoa-flavored diets in the number of urine marks or fecal boli deposited, or in the patterns of deposition.

The control subjects consumed an average of $9.93 \mathrm{~g}$ of the cinnamon diet and $3.33 \mathrm{~g}$ of the cocoa diet, with the cocoa diet constituting $25 \%$ of their consumption. The subjects in the cinnamon demonstration group consumed significantly more of the cinnamon diet and significantly less of the cocoa diet than did both the controls and the subjects in the cocoa demonstration group. This almost exclusive consumption of cinnamon diet is a markedly different pattern of feeding behavior from that exhibited by the controls. The elevated consumption of the cinnamon diet by this group can only be attributed to the powerful influence of the residual deposits left in the enclosure by conspecifics. Thus there is strong evidence for social transmission along chains of animals in the cinnamon demonstration group. The fact that there was no decay in subjects' elevated cinnamon diet consumption right across the transmission chain suggests that information carried by the excretory deposits was available to animals at each step and that it significantly influenced not only their feeding, but also their marking behavior. If no transmission had taken place, the consumption of cinnamon diet by rats in this group would have been expected to decay to that exhibited by the controls within $72 \mathrm{~h}$. The subjects in the cocoa demonstration group showed little evidence for social transmission. Although at the first transmission step they consumed more of the cocoa-flavored diet and less of the cinnamon-flavored diet than did the controls, this preference decayed rapidly along the chain. The decay occurred within $72 \mathrm{~h}$, and it can be explained most parsimoniously in terms of the loss in effectiveness with time of the cues laid down by the original demonstrators. These subjects exhibited a decreasing trend in cocoa diet consumption and an increasing trend in cinnamon diet consumption. Throughout our food-site-marking investigations, naive subjects in the control condition have consistently exhibited a preference for the cinnamon-flavored diet. One interpretation of this difference between the experimental groups is that social transmission may be more stable when it reinforces a prior preference (i.e., for a more palatable diet) than when it conflicts with one. This prior preference may differentially affect the stability of the social transmission of diet preferences among rats in experimental conditions. There is no evidence that demonstrators in the cinnamon demonstration group consumed more diet, urine marked, or defecated more than those in the cocoa demonstration condition, and the differences between the experimental conditions cannot be attributed to differences in demonstrator marking behavior.

Our earlier study (Laland \& Plotkin, 1991) also resulted in a greater total diet consumption among subjects in experimental groups than among controls. This difference 
may be attributable to the greater neophobia experienced by rats confronted with novel foods in an unmarked environment.

\section{EXPERIMENT 3}

Studies of social learning and communication in Norway rats have uncovered a number of processes that facilitate the communication of food preferences among individuals, including following conspecifics to food sites (Galef \& Clarke, 1971) and picking up cues on the breath of conspecifics that have recently eaten (Galef \& Wigmore, 1983). If these processes operate in natural populations, it is unlikely that each will act in isolation. It is thus conceivable that, in a more natural situation, different communication mechanisms may interact to reinforce the social transmission of food preferences.

The goal in this experiment was to investigate whether the unstable transmission of elevated levels of cocoa diet consumption exhibited by subjects in the cocoa demonstration condition in Experiment 2 could be bolstered by the introduction of a second process for the communication of diet preferences. The additional process introduced is the communication of food preferences by way of gustatory cues studied by Galef and coworkers. Galef (1990) reports a series of investigations of the social learning of food preferences among rats via cues on the breath of conspecifics. The principal finding is that a naive rat allowed to interact with a demonstrator conspecific that has recently eaten a novel diet will pick up gustatory cues on the breath of the demonstrator. When subsequently given a choice between the novel diet that the demonstrator consumed and an alternative novel diet, the subject tends to prefer the former. If this process were to act in concert with the social learning of food preferences by excretory marking, the social transmission of a preference for the cocoa-flavored diet among rats should be more stable.

\section{Method}

Design. The design of this experiment was similar to that of Experiment 2, except that the initial demonstrators in all experimental conditions ate the cocoa-flavored diet. This was because the present goal was to investigate whether the transmission of food preferences could be bolstered by the introduction of a second process. Given the stability of transmission of the diet preference in the cinnamon demonstration condition in Experiment 2, it would be pointless to investigate whether the transmission of preferences in this group could be bolstered. There were four conditions, a control and three experimental conditions. For one of the experimental conditions, the excretory marking condition, the procedure was the same as that for the cocoa demonstration group in Experiment 2. For a second experimental condition, the gustatory cues condition, each subject interacted for $30 \mathrm{~min}$ with the animal just removed from the enclosure before being placed in a clean enclosure. The subjects in the third experimental group, the excretory marking and gustatory cues condition, also had the opportunity to acquire gustatory cues directly from their demonstrators, as well as residual cues that previous animals had left in the enclosure. The control condition was the same as in Experiment 2.

Subjects and Apparatus. There were 248 rats of the same kind as in Experiment 1, tested in the same apparatus. There were 6 sub- jects at each step in the experimental groups' chains, and 4 subjects in each (independent) step for the controls. Thus there were 48 animals in each experimental group and 32 controls.

Procedure. The procedure for the control condition was the same as that in Experiment 2, and that for the excretory marking condition the same as that for the cocoa demonstration condition in Experiment 2 . In the gustatory cues condition, we placed 4 rats in the enclosure for $48 \mathrm{~h}$, allowing them to eat from a single bowl, counterbalanced for position, containing the cocoa-flavored diet. Then we removed the demonstrators and placed one of them in a small holding box for 30 min with the 1 st subject. We cleaned the enclosure thoroughly and then placed the subject in it for $24 \mathrm{~h}$ with a choice between the two flavored diets. We then removed this subject, and placed it in a holding box with the next subject in the chain for a 30-min period. This process was repeated for each of the 8 animals in the chain. The procedure was exactly the same for the excretory marking and gustatory cues condition, except that we did not clean out the enclosure after each step in the chain. As in Experiment 2 , we produced a sufficient amount of the flavored diets to allow us to top up the levels in the bowls if necessary, in the same manner as before.

\section{Results and Discussion}

The results are illustrated in Figure 3. The amount of cinnamon diet consumed by subjects varied significantly between the groups $[F(3,144)=15.73, p<.01]$, with subjects in the excretory marking and gustatory cues group consuming significantly less of this diet than did controls (7.48 vs. $11.69 \mathrm{~g}$, respectively) $[F(1,144)=23.1, p<$ .011 . The subjects in the excretory marking and gustatory cues condition also consumed significantly less cinnamon diet than did the subjects in the excretory marking (Newman-Keuls, $W_{4}=2.04, p<.05$ ) and gustatory cues (Newman-Keuls, $W_{2}=1.55, p<.05$ ) groups. The subjects in the gustatory cues and excretory marking groups did not differ significantly from the controls in their cinnamon diet consumption $(M=10.70$ and 12.59$)$. There were no other significant differences in cinnamon diet consumption between the groups. An increasing trend in cinnamon consumption across the chain was found for the excretory marking and gustatory cues condition $[F(1,144)=$ $29.54, p<.01]$, the excretory marking condition $[F(1,144)=19.76, p<.01]$, and the gustatory cues condition $[F(1,144)=7.58, p<.05]$. Although the subjects

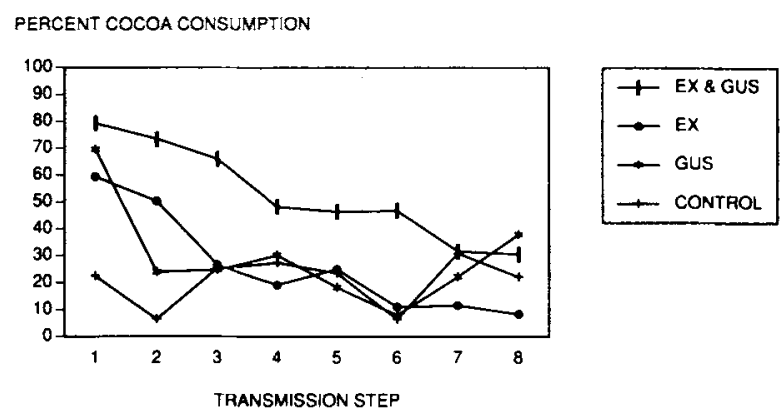

Figure 3. Experiment 3: Percent cocoa-flavored diet consumed by subjects in each group across the transmission chain. EX \& GUS, EX, and GUS are the excretory marking and gustatory cues, excretory marking, and gustatory cues groups, respectively. 
in the excretory marking and the gustatory cues conditions exhibited a reduced consumption of the cinnamon-flavored diet at the first step in the chain, this increased rapidly, and by the third step in the chain, these animals' cinnamon consumption was no different from the controls'. The subjects in the excretory marking and gustatory cues condition showed a more gentle increase in cinnamon diet consumption, and it was only after the seventh step in the chain that these subjects consumed similar levels of this diet as the controls.

There were also significant differences in cocoa diet consumption among the groups $[F(3,144)=14.54, p<$ $.01]$, with the subjects in the excretory marking and gustatory cues group consuming significantly more than the controls $(M=7.88$ vs. $3.06 \mathrm{~g})[F(1,144)=35.35$, $p<.01]$. The subjects in the excretory marking $(M=$ $4.64 \mathrm{~g})$ and gustatory cues $(M=4.23 \mathrm{~g})$ conditions did not consume significantly more of this diet on average than did the subjects in the control condition. The subjects in the excretory marking and gustatory cues condition consumed significantly more cocoa diet than did the subjects in the excretory marking (Newman-Keuls, $W_{2}=1.43, p<.05$ ) and gustatory cues (NewmanKeuls, $\left.W_{3}=1.72, p<.05\right)$ groups. There were no other significant differences in cocoa diet consumption between the groups. A decreasing trend in cocoa diet consumption across the chain was found for the excretory marking and gustatory cues condition $[F(1,144)=$ $18.36, p<.01]$, the excretory marking condition $[F(1,144)=30.11, p<.01]$, and the gustatory cues condition $[F(1,144)=3.56, p=.058]$. Although the subjects in the excretory marking and the gustatory cues conditions exhibited an enhanced consumption of the cocoa-flavored diet at the first step in the chain $[F(1,144)=19.44$ and 19.32 , respectively $]$, this decreased rapidly, and by the third step in the chain these animals' cocoa consumption was no different from that of the controls. The subjects in the excretory marking and gustatory cues condition showed a much more gentle decline in cocoa diet consumption, and it was only after the seventh step in the chain that these subjects consumed similar levels of this diet as did the controls. The results are illustrated in Figure 3, which presents the percentage of total consumption that was cocoa diet, for each group, at each step in the chain.

There were no significant differences in total diet consumption between the groups $[F(3,144)=1.99, p>$ .05].

This experiment provides some evidence that the two social learning processes interacted in such a way as to reinforce the stability of the transmission of food preferences along the chain. Although the subjects in this group exhibited a decline in their levels of diet consumption to levels similar to that of the controls, the introduction of a second social learning process was sufficient to delay this decline. This finding suggests that where a socially transmitted trait is affected by more than one mechanism, it is stablized.

\section{GENERAL DISCUSSION}

The main finding of this study is that enhanced preferences for particular flavored diets can be transmitted along a laboratory chain, providing strong laboratory-based evidence that rats are capable of the social transmission of food preferences. Although many previous laboratory studies have demonstrated the social learning of food preferences by rats, they have not demonstrated its transmission along a chain of animals. The decay in cocoa diet consumption in the cocoa demonstration group of Experiment 2 is a good illustration of how social transmission cannot be assumed from a laboratory demonstration of social learning.

In the two transmission experiments, the control condition demonstrated the level of consumption of each diet when there was no information being transmitted along the chain. When subjects in the experimental groups exhibited a pattern of feeding that was significantly different from that of the controls, it could only be because their behavior was influenced by cues left in the enclosure by previous animals. For the cinnamon demonstration group in Experiment 2, the effect is clear and relatively easy to interpret, since subjects consumed significantly more cinnamon and significantly less cocoa-flavored diet right across the transmission chain. This means that the subjects' behavior throughout was influenced by information contained in excretory deposits left in the enclosure. Since it was demonstrated in Experiment 1 that the excretory cues decayed within 3 days, and since the chain lasted 8 days, the animals at Steps 3-8 in the chain must have been influenced by the marking behavior of subjects other than the original demonstrators. Given the consistency of the marking and feeding behavior exhibited by the subjects in this group, the simplest and most parsimonious explanation for this behavior is that a transmission chain was established in which each animal was influenced by the excretory deposits of previous animals with respect to which diet to eat, how much of each diet to eat, and where to deposit excretory products. The behavior of these animals cannot be explained solely in terms of the more palatable nature of the cinnamon diet, because otherwise they would have exhibited precisely the behavior of the controls. There was no evidence that demonstrators in the cinnamon demonstration group consumed more diet in total, urine marked more, or defecated more than those in the cocoa demonstration condition, and hence the differences between the experimental conditions cannot be attributed to differences in demonstrator marking behavior. The elevated consumption of cinnamon diet by this group is best interpreted as resulting from an interaction between socially acquired information carried by the deposits and a prior preference for cinnamon, possibly resulting from its more palatable nature. An interaction of this type also explains the results of the cocoa demonstration group: here the socially transmitted information initially conflicted with the prior preference for cinnamon. As the original cues decay, subjects are increasingly inclined to eat, and 
subsequently mark, the cinnamon diet. Eventually the cues surrounding the cocoa diet decay, leaving only the cinnamon diet marked. These animals thus eventually behave like those in the cinnamon demonstration group. The significance of the findings of this experiment is that previously learned, experiential, or "innate" predispositions may significantly affect the stability of socially transmitted traits. If such predispositions do have a genetic basis, they are comparable to Lumsden and Wilson's (1981) "epigenetic rules," which influence the probability of individuals' adopting a cultural variant.

Although laboratory studies have uncovered a number of mechanisms that can result in the social learning of food preferences among rats, these are unlikely to operate independently in natural populations. Experiment $3 \mathrm{dem}$ onstrated that different social learning processes can interact to reinforce the stability of socially transmitted food preferences. The transmission of a diet preference was found to be much more stable when two social learning mechanisms operated together than when either operated in isolation. This finding suggests that when a particular socially transmitted trait is mediated by more than one process, it is likely to be more stable. It also means that a failure to find social learning and transmission in the laboratory, where only one mechanism is investigated, does not necessarily demonstrate that transmission is unlikely under more natural conditions, where a host of mechanisms may operate together. This study illustrates the sensitivity and complexity of animal social learning and transmission.

\section{REFERENCES}

Brown, R. E. (1985). The rodents II. In R. E. Brown \& D. W. Macdonald (Eds.), Social odours in mammals (Vol. 2, pp. 345-457). Oxford: Oxford University Press, Clarendon Press.

Calmoun, J. B. (1962). The ecology and sociology of the Norway rat (Public Health Publication No. 1008). Bethesda, MD: U.S. Department of Health.

Curio, E., Ernst, U., \& VIETH, W. (1978). The adaptive significance of avian mobbing II. Cultural transmission of enemy recognition in blackbirds: Effectiveness and some constraints. Zeitschrift fur Tierpsychologie, 48, 184-202.

GALEF, B. G., JR. (1988). Communication of information concerning distant diets in a social, central place foraging species (Rattus norvegicus). In T. R. Zentall \& B. G. Galef, Jr. (Eds.), Social learning (pp. 119-139). Hillsdale, NJ: Erlbaum.

GALEF, B. G., JR. (1990). An adaptationist perspective on social learning, social feeding, and social foraging in Norway rats. In D. A. Dewsbury (Ed.), Contemporary issues in comparative psychology (pp. 5579). Sunderland, MA: Sinauer.

GALEF, B. G., JR., BECK, M. (1985). Aversive and attractive marking of toxic and safe foods by Norway rats. Behavioral \& Neural Biology, 43, 298-310.

GAlEF, B. G., JR., \& Clarke, M. M. (1971). Social factors in the poison avoidance of feeding behaviour of wild and domesticated rat pups. Journal of Comparative \& Physiological Psychology, 75, 341-357.

GALEF, B. G., JR., \& HEIBER, L. (1976). Role of residual olfactory cues in the determination of feeding site selection and exploration patterns of domestic rats. Joumal of Comparative \& Physiological Psychology, 90, 727-739.

GaLEF, B. G., JR., \& Wigmore, S. W. (1983). Transfer of information concerning distant foods: A laboratory investigation of the "information-centre" hypothesis. Animal Behaviour, 31, 748-758.

Gandolfi, G., \& PARISI, V. (1973). Ethological aspects of predation by rats Rattus norvegicus (Berkenhout), on bivalves Unio pictorum L. and Cerastoderma lamarcki (Reeve). Bollettino di Zoologia, 40, 69-74.

JoNES, R. B., \& NowELL, N. W. (1977). The emotional responses of male laboratory mice to various urinary odors. Behaviour \& Biology, 19, 98-107.

Laland, K. N., \& Plotkin, H. C. (1990). Social learning and social transmission of foraging information in Norway rats (Rattus norvegicus). Animal Learning \& Behavior, 18, 246-251.

Laland, K. N., \& PlotKin, H. C. (1991). Excretory deposits surrounding food sites facilitate social learning of food preferences in Norway rats. Animal Behaviour, 41, 997-1005.

LuMSDEN, C. J., \& Wison, E. O. (1981). Genes, mind and culture. Cambridge, MA: Harvard University Press.

LYDELL, K., \& DoTY, R. L. (1972). Male rat odor preferences for female urine as a function of sexual experience, urine age, and urine source. Hormones \& Behavior, 3, 205-212.

STEINIGER, F. von (1950). Über Duftmarkierung der Wanderratte. Zeitschrift für Angewandte Zoologie, 38, 357-361.

(Manuscript received April 12, 1991; revision accepted for publication June 20, 1992.) 